\title{
A Young female with patent foramen ovale and cryptogenic stroke
}

\author{
Xinhua Xü ${ }^{1 *}$, Songbai $\mathrm{Du}^{2 *}$ and Xuguang Qin ${ }^{1 *}$ \\ ${ }^{1}$ Department of Cardiology, First Affiliated Hospital of Tsinghua University, Beijing 100016, China \\ ${ }^{2}$ Department of Cardiology, Nanbu County's Hospital of Sichuan Province, China \\ \#Contributed equally to this work
}

\section{Introduction}

The definition of patent foramen ovale was first raised by Leonardi Botali [1] in the year 1955. Patent foramen is a residual part of the fetal circulation system. It is an open area in the center of the inter-atrial septum. After birth, when the systemic circulation has been formed, the pressure in the left atrium increases gradually leading to a functional closing. Generally speaking, it's closed during the first year after birth. If the child's patent foramen is still not closed until he's more than 3 years old, this phenomenon is called patent foramen ovale (PFO). About $27 \%$ of general population, the patent foramen is not closed completely, leaving small cracks [2]. Generally small PFO $(<2 \mathrm{~mm})$, which has no hemodynamic changes, does not cause contradiction embolism mostly. However, in rare cases, due to the emotional changes, the changes of blood pressure, acute severe cough, doing Valsalva gesture, when the pressure in the right heart increases suddenly, which may provoke the right to left shunt. In the study of Steiner et al. [3] showed that medium PFO ( $\geq 2 \mathrm{~mm}$ ) and large PFO ( $\geq 4 \mathrm{~mm}$ ) have higher frequencies to cause cerebral infarction (CI) than small or no PFO. When a person has ASA and PFO, the blood flow nearby becomes irregular, with embolus formed easily. Then embolus from the right heart system travels through the patent foramen ovale into the left heart, flows from the aorta to the systematic system, leading to cerebral infarction

\section{Case}

This is a case of a 24-year-old female who used to be in good health without taking oral contraceptives ever before. She was presented to the local hospital with numbness and weakness on the right side on 22 April 2014. Four and a half hours before, she had got a great anger over her little girl. The magnetic resonance imaging (MRI) of her head showed the left thalamus infarction and the left cerebellar infarction (Figure 1). Comprehensive stroke work-ups including the blood tests, the magnetic resonance angiography (MRA) of her head (Figure 2), the Holter monitor, the ultrasound of the carotid arteries and the lower limbs' vessels did not reveal any straightforward causes for stroke except for a patent foramen ovale (PFO) which had a left-to-right shunt described on TTE. No further abnormalities were reported. Anti-coagulation with a vitamin $\mathrm{K}$ antagonist named warfarin was taken ever since. On $9^{\text {th }}$ June 2014, she with her family referred to our hospital for percutaneous PFO closure for secondary stroke prevention. The TTE taken before the PFO closure operation showed that there was a medium PFO (2 $\mathrm{mm}$ ) existed with a right-to-left shunt at the atrial level (Figure 3). On $12^{\text {th }}$ June 2014, she had an operation of percutaneous PFO closure. The procedure performed as following: The patient had a right femoral vein
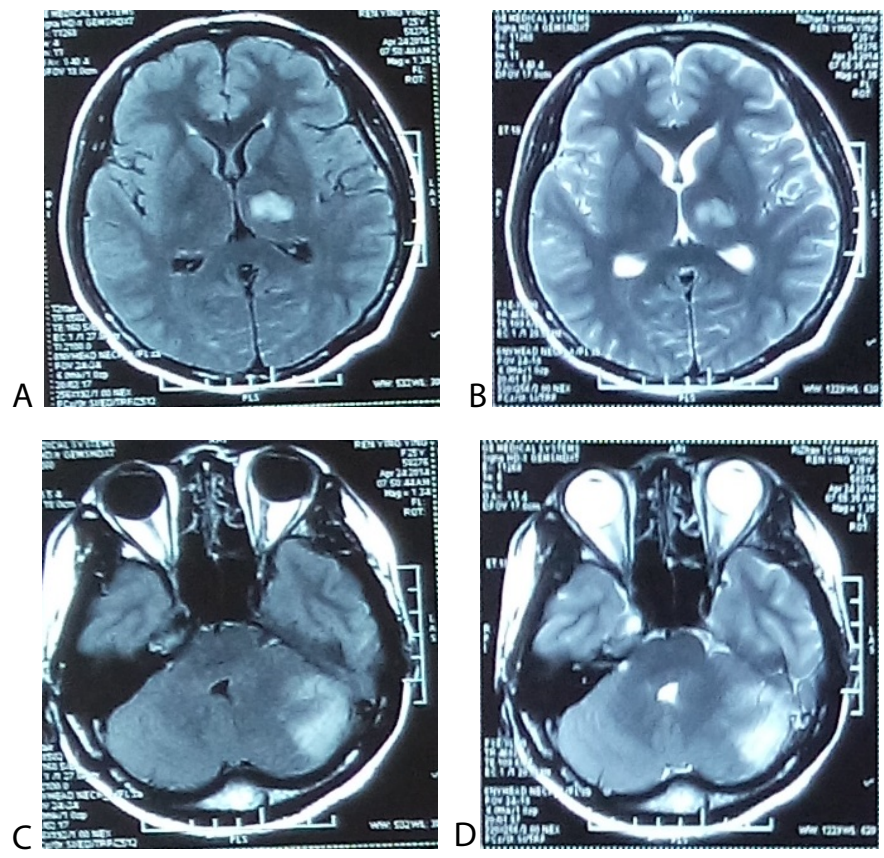

Figure 1. A) The magnetic resonance imaging brain examination (axial view MRI-DWI image): demonstrating left thalamus infarction. B) The magnetic resonance imaging brain examination (axial view MRI-T2weighted image): demonstrating left thalamus infarction. C) The magnetic resonance imaging brain examination (axial view MRI-DWI image): demonstrating left cerebellar infarction. D) The magnetic resonance imaging brain examination (axial view MRI-T2weighted image): demonstrating left cerebellar infarction

puncture, under the condition of oxygen and local anesthesia; Then she experienced a right cardiac catheterization examination with a $5 \mathrm{~F}$ catheter line; Under the X-ray and ultrasonic monitoring, a $18 / 18 \mathrm{~mm}$ diameter PFO closure was implanted currently; During the operation, heparin was used with a dose of $100 \mathrm{iu} / \mathrm{kg}$. After a conventional hospital course, the patient was discharged on the forth post-operative day in a quite good condition. Postoperative echocardiography depicted that the closure was working well with no obvious shunt (Figure 4).

*Correspondence to: Xuguang Qin, Department of Cardiology, First Affiliated Hospital of Tsinghua University, Beijing 100016, China, E-mail: qin_xuguang0712@163.com

Key words: patent foramen ovale (PFO), cryptogenic stroke (CS), young female

Received: May 7, 2018; Accepted: May 24, 2018; Published: May 29, 2018 


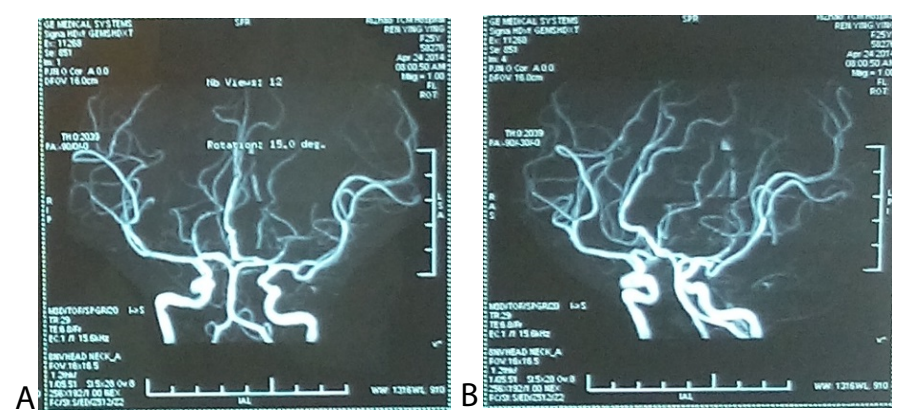

Figure 2. A, B: The magnetic resonance angiography (MRA) brain examination is in good condition

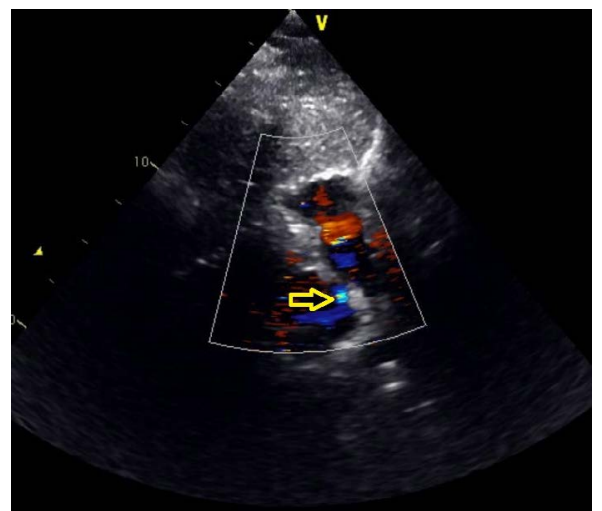

Figure 3. The TTE taken before the PFO closure operation showed that there was a medium PFO existed with a right-to-left shunt at the atrial level (As shown in figure yellow arrow)
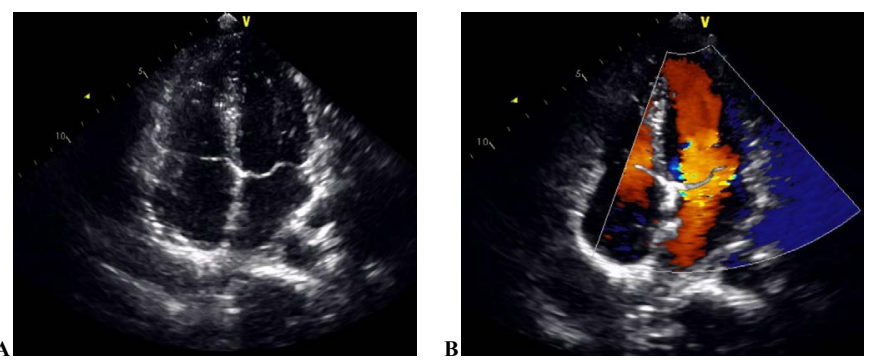

Figure 4. A, B: Postoperative echocardiography depicted that the closure was working well with no obvious shunt

\section{Discussion}

\section{The diagnosis of PFO}

A) Trans-thoracic echocardiography (TTE): It is a popular method among patients to make the diagnosis. However, it generally cannot find out direct positive signs. B) Trans-esophageal echocardiography (TEE): It is the gold standard in the diagnosis of patent foramen ovale. C) Trans-cranial Doppler (TCD): It can detect the signals of embolus from $\mathrm{PFO}$ in the brain blood circulation.

\section{The treatment of PFO with cryptogenic stroke}

It includes drug treatment and surgical treatment: Drug therapy including anti-coagulation and anti-platelet therapy. Surgical treatment includes open chest cardiac surgery and percutaneous PFO closure. Percutaneous PFO closure is currently the most effective method of surgical treatment. Implantation of a double-umbrella device in the patent foramen ovale are achieved in all without serious procedural complications. Trans-catheter closure of a patent foramen ovale may reduce the risk of recurrence.
Basing on the points made above, the mechanism of patients with PFO with cryptogenic stroke is still controversial. Some scholars think that paradoxical cerebral embolism is its possible mechanisms. The sudden rise of the pulmonary artery pressure will raise the pressure of the right atrium. When the pressure in the right atrium is greater than the left, the emboli formed in the right will run into the left with blood flow [4]. On this mechanism, the emboli from the venous system can cause cerebral infarction. This principle may also make some points in young people with left to right shunt who are also suffered from cryptogenic stroke. In general, for most of the people with PFO, there is no right-to left shunt, however, when the pressure of the right atrium is increasing suddenly, the emboli can flow from the right atrium to the left. In our case, the MRA and vascular ultrasound of the patient does not show clear left heart system thrombosis. So where does the embolus come? Some scholars believe that the formation and thrombolysis of emboli in the body is in a dynamic process, they are in a relatively stable state. So, it's not too difficult to explain the source of thrombosis which come from the blood system. The TTE of the patient shows a right to left shunt at the atrial level (Figure 3). Its mechanism of cryptogenic stroke may be that the great anger may cause sudden rises of the pulmonary system, which causes the pressure of the right atrium greater than the left. In this way, the embolus may travel from the right atrium to the left, causing cerebral infarction to the patient. Other scholars hold the opinions that the patients with PFO and cryptogenic stroke may have the following mechanism: 1 . Atrial arrhythmia might be caused by PFO, which can result in atrial thrombosis; 2 . The stagnant blood through within the patent foramen ovale may lead to thrombosis; 3. The high blood condensation state may be caused by PFO [5]. For this patient, the blood chemical tests and Holter monitor do not make any certain evidence, so the causes mentioned above don't take into consideration. And some scholars think that the patients with PFO and cerebral infarction tend to have posterior circulation infarction [5]. The MRI-DWI of our patient does show that the left thalamus infarction (Figure 1A) and left cerebellar infarction (Figure 1C). It is in relation with the blood supply zone of deep perforators which is from the posterior circulation artery. Basing on all the evidences mentioned above, we can conclude that the patient with cerebral infarction may be caused by posterior circulation thromboembolism. In recent years, the incidence reported of youth cerebral infarction is rising year by year. However, once the youth have cryptogenic cerebral infarction, they should see a doctor in time in order to get a better recovery. This young female is only 24 years old, because of her good health in usual. After regular treatment, her body restore well, with no obvious sequelae left.

This case emphasizes a 24-year-old married female with PFO and cryptogenic stroke. We report this case, in order to raise the reconstruction of the physicians both in the departments of cardiology and neurology to pay more attention to the young patients with PFO and $\mathrm{CI}$ in our society. At the same time, through this case, we can know more about the typical clinical symptoms of young people with PFO and cryptogenic stroke. In order to explore the convenient detections to make current diagnosis of patients, we look forward to figuring out the suitable treatment in the following period. Keeping in good health, one need to calm down, do more exercise and be on a diet.

\section{References}

1. Carerj L (1955) Leonardo Botallo, the foramen ovale and the ductus arteriosus. Minerva Med 46: Varia, 789-795. [Crossref] 
2. Hagen PT, Scholz DG, Edwards WD (1984) Incidence and size of patent foramen ovale during the first 10 decades of life: an autopsy study of 965 normal hearts. Mayo Clin Proc 59: 17-20. [Crossref]

3. Stenier MM, Di Tullio MR, Rundek T, Gan R, Chen X, et al. (1998) Patent foramen ovale size and embolic brain imaging findings among patients with ischemic stroke. Stroke 29: 944-948. [Crossref]
4. Drighil A, El Mosalami H, Elbadaoui N, Chraibi S, Bennis A (2007) Patent foramen ovale: a new disease? Int $J$ Cardiol 122: 1-9. [Crossref]

5. Jauss M, Wessels T, Trittmacher S, Allendoerfer J, Kaps M (2006) Embolic lesion pattern in stroke patients with patent foramen ovale compared with patients lacking an embolic source. Stroke 37: 2159-2161.

Copyright: (C2018 Xu X. This is an open-access article distributed under the terms of the Creative Commons Attribution License, which permits unrestricted use, distribution, and reproduction in any medium, provided the original author and source are credited. 